\title{
Stability and Performance Characteristics of a Digital Positron Lifetime Spectrometer
}

\author{
R. Aavikko, K. Rytsölä, J. Nissilä and K. SaArinen \\ Laboratory of Physics, Helsinki University of Technology \\ P.O. Box 1100, 02015 HUT, Espoo, Finland
}

We summarize the setup and performance tests of a digital positron lifetime spectrometer presently in routine use at HUT/Laboratory of Physics. The performance of the spectrometer is found to be equal to or better than that of an analog spectrometer with similar detectors. We present also results on measuring the stability of the system, which is found to be similar to that of analog spectrometers: variation in the time-zero of the spectrometer is found to be $\approx 10 \mathrm{ps} /$ week. We suggest that the remaining instabilities of the spectrometers originate primarily from the detectors.

PACS numbers: 06.60.Jn, 07.05.Hd, 78.70.Bj, 07.85.Nc

\section{Introduction}

The detector signals of a positron lifetime spectrometer have conventionally been processed with analog electronics composed of nuclear instrumentation modules (CFDs, TAC, MCA). The advent of fast ( $\geq 1 \mathrm{GS} / \mathrm{s}$, GS = gigasamples) ADCs and increase in the available computing power has allowed replacing the analog electronics chain with a digitizer and a computer. The pulse processing including timing, energy windowing, histogramming etc. is then performed with software directly from the digitized detector pulses. Digital positron lifetime spectrometers have been realized earlier with fast digital oscilloscopes $[1,2]$. Excellent resolution $(\approx 150 \mathrm{ps})$ has been obtained with optimized detectors, and possibility of capturing both $511 \mathrm{keV}$ annihilation $\gamma$-quanta has been demonstrated [2]. The spectrometer setup with a digitizer card at Helsinki University of Technology, Laboratory of Physics has been reported and its performance analyzed in [3, 4]. The results show that the performance of the digital spectrometer is equal to or 
better than that of the analog spectrometer. Especially the accuracy with small detector pulse amplitudes, walk performance and linearity are found to exceed the characteristics of analog spectrometers. The data throughput capacity obtained with a digitizer card is higher than the count rates of typical lifetime measurements. In this paper we summarize the performance results and report new ones on the medium term (day-week) stability of the spectrometer.

\section{Setup and methods}

The block diagram and a photograph of the digital positron lifetime spectrometer are shown in Fig. 1. Two fast scintillation detectors capture the $\gamma$-quanta emitted at the birth and the annihilation of the positron. The anode pulses from the photomultiplier tubes (PMT) are fed into a combiner and led via a single cable to the digitizer. The anode pulse of the stop detector is delayed with $\approx 50 \mathrm{~ns}$ cable delay in order to avoid the piling up of the two anode pulses. Here the scintillation detectors are composed of fast plastic cylindrical scintillators $\left(\phi 30 \times 20 \mathrm{~mm}^{3}\right.$ NE-111) and XP2020 photomultipliers (Photonis).
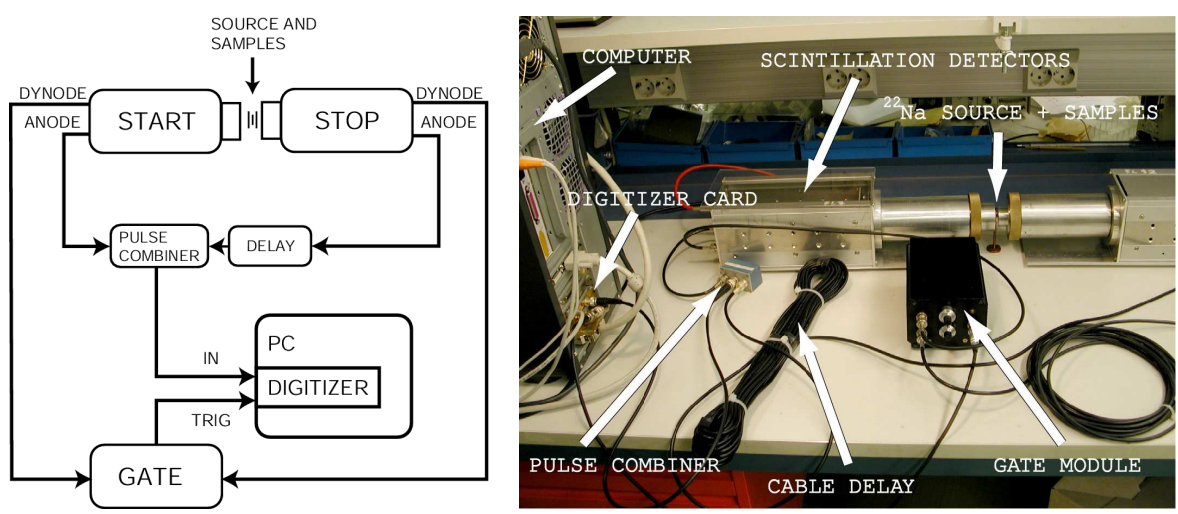

Fig. 1. A block diagram of the digital positron lifetime spectrometer [3] and a photograph of the setup. START and STOP are fast scintillation detectors, GATE - a triggering module for detecting coincidences, DELAY - a $\approx 50$ ns cable delay and PULSE COMBINER - an impedance matched power splitter.

The digitizer used in the spectrometer is an 8-bit digitizer card DP210 by Acqiris connected to the PCI-bus of the measurement computer. The bandwidth of the card is $500 \mathrm{MHz}$ and its maximum sampling rate $2 \mathrm{GS} / \mathrm{s}$.

To avoid transferring most of the non-coincident pulses to the computer (ratio of coincidences to singles is only a couple of per cent), a "home made" gate module is used to trigger the digitizer in case of start-stop coincidence. The module consists of two lower level discriminators and a coincidence circuit realized with fast comparators and two D flip-flops. 
The computer of the spectrometer has dual AMD Athlon MP 1900+ processors, 1 GB memory and $\approx 100 \mathrm{~GB}$ hard disk space. A computer program, written in $\mathrm{C}++$, simultaneously controls the digitizer and analyzes the data. This solution reduces the dead time in a usual positron lifetime measurement to a negligible level $(<1 \%)$, where it is determined by the transfer time of the data.

The data analysis of a single sweep (digitized start-stop coincidence) consists of (i) checking that the sweep contains exactly two pulses (one is not sufficient and a third pulse might interfere with the timing), (ii) checking that the anode pulses fit in energy (amplitude) windows, (iii) extraction of the time information from the digitized pulse pairs, (iv) updating the lifetime histogram with the time interval. The extraction of the timing instants from the digitized anode pulses (iii) is performed by constant fraction (CF) timing. For this we use fitting a smoothing spline to the leading edge of the anode pulse and determining the CF timing instant from the fitted curve. Histogramming of the time interval is straightforward and the bin width (i.e. time channel width, typically 10-30 ps) can be arbitrarily chosen.

\section{Performance of the spectrometer}

Generally the time resolution of a well tuned positron lifetime spectrometer is dominated by the detectors, especially the (sizes of) scintillators and the used energy windows. Thus the accuracy of the digital part of the spectrometer was investigated by comparing it to an analog spectrometer. To assure reliable comparison, the same events were handled by both systems (this way e.g. the energy windows of the two systems are equal). This was accomplished by dividing the anode pulses with splitters to the discriminators of the analog spectrometer and the pulse combiner of the digital one. The data acquired with the two systems are shown in the left part of Fig. 2. No difference between the two spectra can be noticed, suggesting that the contribution of the electronics is negligible in both cases.

The right part of Fig. 2 presents a positron lifetime spectrum in bulk SiC measured with the digital spectrometer. After subtraction of the source components, a single exponential convoluted with a Gaussian resolution function fits the data well. The fitted lifetime equals $146.7 \pm 0.2 \mathrm{ps}$ in agreement with previous experiments [5]. The time resolution obtained from the fit is $222 \mathrm{ps}$ (FWHM, 50\% ${ }^{22} \mathrm{Na}$ energy windows).

The attainable count rate is an important feature for a lifetime spectrometer. In a test of data transfer rate using a pulser, up to 30,000 unprocessed events per second could be recorded to computer main memory, whereas the computing power of the present computer limits the maximum rate of analyzed events to $3,0001 / \mathrm{s}$. Both values are much higher than typical count rates in normal positron lifetime experiments ( $\approx 2001 / \mathrm{s}$ and limited by the detectors and the source activity). 

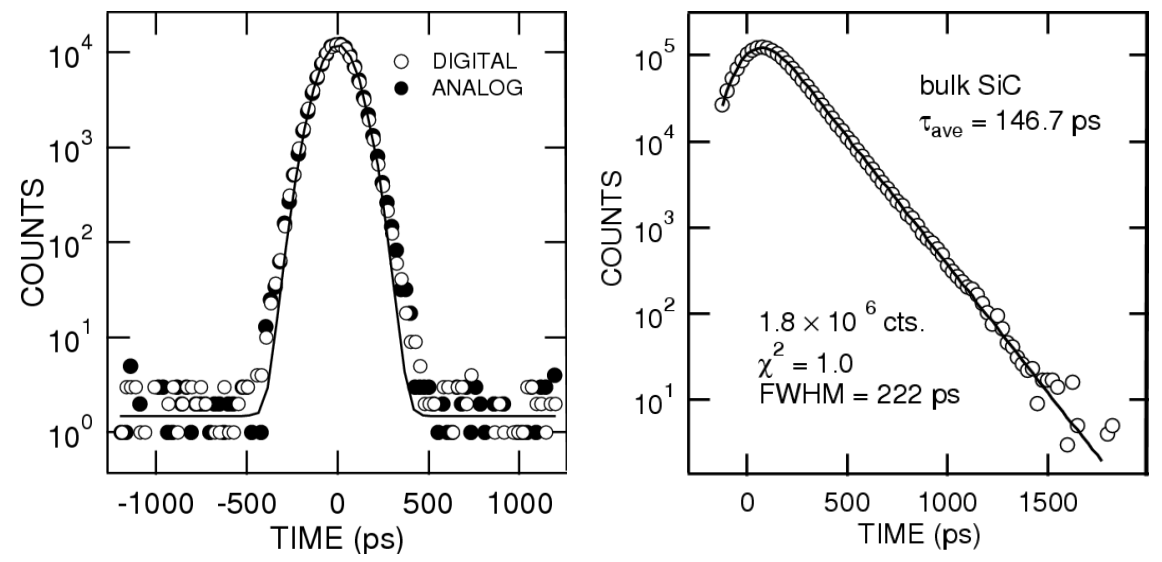

Fig. 2. The left part shows resolution functions obtained from the same pulses with analog and digital timing electronics [3]. The FWHM $=212 \mathrm{ps}$ for both sets of data (energy windows are $\approx 60 \%{ }^{60} \mathrm{Co}$, set by the analog discriminators). The right part shows a positron lifetime spectrum in bulk $\mathrm{SiC}$ (source- and background corrected).

\section{Medium-term stability of the spectrometer}

The stability of a lifetime spectrometer can be studied by measuring the position of the coincidence peak, as changes in both the true time zero

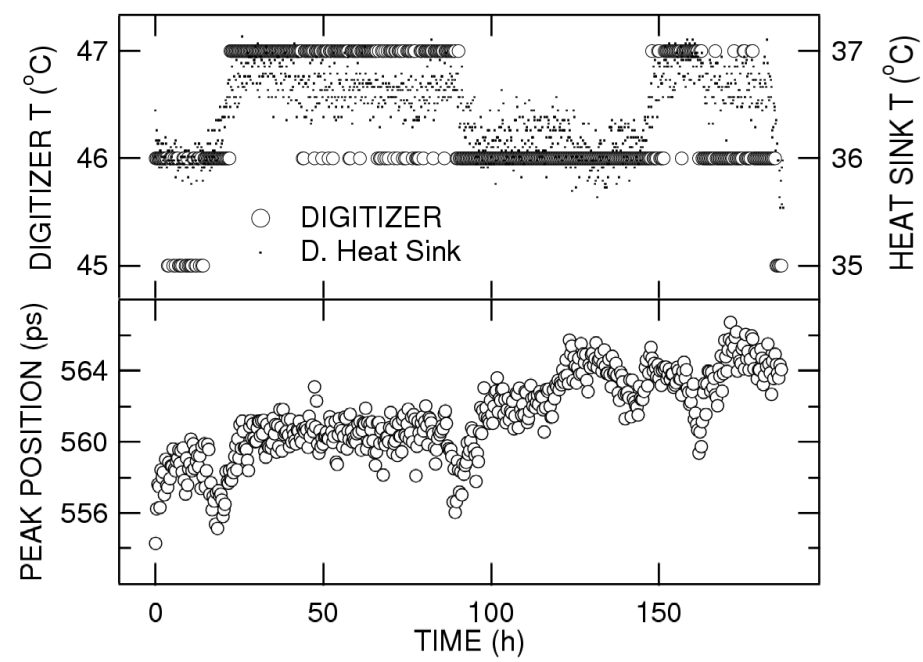

Fig. 3. Digitizer stability measurement. The peak positions have been obtained by measuring $\approx 110000$ pulses from a ${ }^{60} \mathrm{Co}$ source. The temperatures are presented as reported by the digitizer (offset of the thermometer of the particular digitizer is apparently off by $\left.\approx 10^{\circ} \mathrm{C}[6]\right)$ and as measured with a thermocouple connected to the heat sink of the digitizer. The magnitude of the drift of the system is $\approx 10 \mathrm{ps} /$ week, which is typical value also of analog spectrometers. 
("offset") and in the time conversion ("gain") affect it. We measured repeatedly $\approx 110000$ count ${ }^{60} \mathrm{Co}$ spectra for more than one week. The positions of the peaks were then determined by fitting Gaussian functions. The spectrometer was located in an airconditioned laboratory, whose temperature was $21-22^{\circ} \mathrm{C}$. The results of the measurements are shown in Fig. 3. As can be seen from the figure, the magnitude of the time-zero drift of the spectrometer is $\approx 10 \mathrm{ps} /$ week, which is typical also of analog spectrometers [7]. The drift of the analog spectrometers has been attributed primarily to the detectors and CFDs [8]. The similarity in the instabilities of the two types of spectrometers with totally different timing systems suggests that the instabilities originate from the detectors.

One candidate for causing the drift is a change in the ambient temperature, also presented in Fig. 3. The figure shows that the temperature and the drift of the spectrometer do not correlate strongly. Still, some similarities can be found - the temperature change coincidences with change in time-zero at least near $20 \mathrm{~h}$ and $90 \mathrm{~h}$ instants. Large temperature variations do cause drift - we have observed up to 20 ps changes in the time-zero, clearly due to temperature changes when using the spectrometer in a non-airconditioned room.

We tried to improve the stability of the spectrometer by using a cable delay (see Fig. 1) with low temperature coefficient (Andrew FAJ1-50A, signal propagation temperature coefficient $-0.03 \mathrm{ps} /(\mathrm{m} \mathrm{K}))$ instead of normal coaxial cable $(-0.25 \mathrm{ps} /(\mathrm{m} \mathrm{K}))[9]$ (length of the delay cable is $\approx 10 \mathrm{~m})$. No clear difference in the drift behavior using the two cable delays could be seen.

\section{Conclusions}

We have summarized the setup and performance results of a digital positron lifetime spectrometer which has been in routine use for semiconductor research at Helsinki University of Technology, Laboratory of Physics for more than one year.

The results show that the performance of the digital system is equal to or better than that of the analog. The time resolution of the system $(\approx 220 \mathrm{ps})$ is similar to that achieved with analog devices, and is primarily determined by the scintillation detectors. Also, the data throughput capacity of the spectrometer is an order of magnitude higher than the count rates of typical $\mathrm{e}^{+}$lifetime measurements.

The stability of the digital system was found to be similar to that of an analog spectrometer: the variation in the time-zero of the system is $\approx 10 \mathrm{ps} /$ week and it does not depend strongly on the temperature changes. We suggest that the main source of the variation arises from the detectors.

Digital processing of detector pulses allows simple construction of positron lifetime spectrometer, and various offline corrections to the data. Together with the performance improvements and lower price these present significant advantages over the analog system. 


\section{References}

[1] K. Rytsölä, J. Nissilä, K. Kokkonen, A. Laakso, R. Aavikko, K. Saarinen, Appl. Surf. Sci. 194, 260 (2002).

[2] H. Saito, Y. Nagashima, T. Kurihara, T. Hyodo, Nucl. Instrum. Methods Phys. Res. A 487, 612 (2002).

[3] J. Nissilä, K. Rytsölä, R. Aavikko, A. Laakso, K. Saarinen, P. Hautojärvi, Nucl. Instrum. Methods Phys. Res. A, accepted for publication.

[4] R. Aavikko, K. Rytsölä, J. Nissilä, Mater. Sci. Forum 445-446, 462 (2004).

[5] R. Krause-Rehberg, H.S. Leipner, Positron Annihilation in Semiconductors, Springer, Heidelberg 1999.

[6] A. Rothenberg, private communication, Acqiris, 2004.

[7] J. Nissilä, M. Karppinen, K. Rytsölä, J. Oila, K. Saarinen, P. Hautojärvi, Nucl. Instrum. Methods Phys. Res. A 466, 527 (2001).

[8] I.K. MacKenzie, in: Proc. Int. School of Physics "Enrico Fermi", Course LXXXIII, Varenna (Italy) 1981, Eds. W. Brandt, A. Dupasquier, North-Holland, Amsterdam 1983, p. 220.

[9] T. Kohama, Temperature Dependence of Propagation Time in Andrew's Coaxial Cable, Technical report, Phoenix group, Hiroshima University, 1996. 